\title{
Interface-Induced Plasmon Nonhomogeneity in Nanostructured Metal-Dielectric Planar Metamaterial
}

\author{
A. I. Kovalev, ${ }^{1}$ D. L. Wainstein, ${ }^{1}$ A. Yu. Rashkovskiy, ${ }^{1,2}$ R. Gago, ${ }^{3}$ F. Soldera, ${ }^{4}$ \\ J. L. Endrino, ${ }^{5}$ and G. S. Fox-Rabinovich ${ }^{6}$ \\ ${ }^{1}$ Surface Phenomena Researches Group, CNIICHERMET, 2nd Baumanskaya Street 9/23, Office 475, Moscow 105005, Russia \\ ${ }^{2}$ National University of Science and Technology (MISiS), Leninskiy Prospekt 4, Moscow 119049, Russia \\ ${ }^{3}$ Instituto de Ciencia de Materiales de Madrid, Consejo Superior de Investigaciones Científicas, 28049 Madrid, Spain \\ ${ }^{4}$ Department of Materials Science \& Engineering, Saarland University, 66123 Saarbruecken, Germany \\ ${ }^{5}$ Manufacturing and Materials Department, School of Aerospace, Transport and Manufacturing (SATM), Cranfield University, \\ College Road, Cranfield, Bedfordshire MK43 OAL, UK \\ ${ }^{6}$ Department of Mechanical Engineering, McMaster University, 1280 Main Street West, Hamilton, ON, Canada L8S 4L7
}

Correspondence should be addressed to G. S. Fox-Rabinovich; gfox@mcmaster.ca

Received 13 November 2014; Accepted 17 February 2015

Academic Editor: Nay Ming Huang

Copyright (C) 2015 A. I. Kovalev et al. This is an open access article distributed under the Creative Commons Attribution License, which permits unrestricted use, distribution, and reproduction in any medium, provided the original work is properly cited.

Transformations of the electronic structure in thin silver layers in metal-dielectric (TiAlN/Ag) multilayer nanocomposite were investigated by a set of electron spectroscopy techniques. Localization of the electronic states in the valence band and reduction of electron concentration in the conduction band was observed. This led to decreasing metallic properties of silver in the thin films. A critical layer thickness of $23.5 \mathrm{~nm}$ associated with the development of quantum effects was determined by X-ray photoelectron spectroscopy. Scanning Auger electron microscopy of characteristic energy losses provided images of plasmon localization in the Ag layers. The nonuniformity of plasmon intensities distribution near the metal-nitride interfaces was assessed experimentally.

\section{Introduction}

Thin-film growth based on plasma technologies is well developed, permitting very complex structures such as multilayers with thicknesses control of individual layers from a few to hundreds of nanometers and the total thickness of the coating of up to tens of microns. These multilayer structures present a cross-section with modulated distribution throughout the coating. Such planar nanolaminate coatings have good stability under a wide range of temperatures and mechanical loads [1].

Localization of plasmon oscillations in solids is a basis for the development of newest prospective nanomaterials. Among them, multilayer metal-dielectric coatings possess unique physical properties and could find many applications as plasmonic metamaterials in a wide range of industries: electronics, power generation, mechanical engineering, and so forth. They have a wide range of useful properties determined by the characteristic response of artificial nanostructure at interaction with an electromagnetic field [2]. The intensity of electrical, magnetic, or optical response depends on matching the external radiation wavelength with the characteristic dimensions of the nanostructure element which determine resonance conditions. Primary radiation can be amplified up to $10^{2}-10^{4}$ times [3]. Such plasmonbased planar nanocomposites have the potential to exhibit the same response as resonator-based metamaterials [4-8]. Plasmonic waveguides can also potentially serve as basic elements of fully three-dimensional metamaterials when arranged in stacked geometries [9]. Anisotropy of plasmon wave propagation in metal-dielectric nanolaminates could be used for strong amplification of a signal in waveguides and resonators as well as its significant attenuation. Significant reduction of heat conductivity in the thin film composite was found, [10] opening prospects of application of such structures as impulse heating protectors. Control over spatial 
plasmon propagation and its coupling at metal-insulator interfaces is a key problem for 3D functional nanostructure design.

Up to the present time, images of plasmon density in metamaterials were built from computer modeling only [11]. This paper presents experimentally determined transformations of electronic structure in the multilayer nanolaminate TiAlN/Ag coatings occurring with layer thickness decreasing. Electron spectroscopy and experimental visualization of plasmon densities by microscopy of characteristic losses were used for these investigations.

\section{Materials and Methods}

Titanium aluminum nitride (TiAlN) monolithic thin films and multilayer TiAlN/Ag coatings were deposited on $\mathrm{Si}$ substrates by direct-current magnetron cosputtering (DCMS) using individual $\mathrm{Ti}_{50} \mathrm{Al}_{50}(99,95 \%$ purity) and $\mathrm{Ag}$ (99,95\% purity) targets. In particular, $\sim 1 \mu \mathrm{m}$ thick coatings were deposited as follows: $150 \mathrm{~nm} / 150 \mathrm{~nm} \times 3$ bilayers (3BL); $20 \mathrm{~nm} / 200 \mathrm{~nm} \times 12$ bilayers (12BL); $150 \mathrm{~nm} / 15 \mathrm{~nm} \times 6$ bilayers $(6 \mathrm{BL}) ; 15 \mathrm{~nm} / 15 \mathrm{~nm} \times 35$ bilayers $(35 \mathrm{BL})$; and $10 \mathrm{~nm} / 5 \mathrm{~nm} \times$ 33 bilayers $(33 \mathrm{BL})$. The crystalline structure of the samples was examined by glancing-angle X-ray diffraction (GIXRD) measurements using a D5000 (Bruker AXS) diffractometer with $\mathrm{Cu}$ Ka radiation $(\lambda=1.5418 \AA)$. The data was collected with an incidence angle of $1^{\circ}$ within the scattering range, $2 \theta$, of $30-80^{\circ}$. In addition, the multilayer structure was imaged by scanning transmission electron microscopy (STEM). For this purpose, cross-sectional specimen was prepared by slicing the samples in a focused ion beam and scanning electron microscope (SEM) dual beam system (FEI Helios 600) by using the "in situ" lift-out technique. The foil was thinned to electron transparency, first, with an acceleration voltage of $30 \mathrm{kV}$ and, later, with $5 \mathrm{kV}$ (for at least $2 \mathrm{~min}$ in each side) to minimize any possible preparation artifacts.

The electron spectroscopy investigations were carried out on the electron spectrometer ESCALAB MK2 (VG) with LEG200 scanning electrons gun $\left(E_{0}=0.1-4 \mathrm{keV}\right.$, spot diameter $200 \mathrm{~nm})$, Al $K_{\alpha}$ X-ray monochromatic source $(h v=$ $1486.6 \mathrm{eV}$, spot $1 \times 8 \mathrm{~mm}$ ), and EMU50 monochromatic low energy electrons source at vacuum $1 \times 10^{-8} \mathrm{~Pa}$. The $\mathrm{C} 1 \mathrm{~s}$ XPS peak $(\mathrm{BE}=285.0 \mathrm{eV})$ was used as an internal reference line to accurately determine the positions of other spectral lines. The spectra acquisition parameters were selected in order to provide the best energy resolution and signal to noise ratio.

The sample surface was cleaned by $\mathrm{Ar}^{+}$ions etching in the preparation chamber of the spectrometer at argon pressure $10^{-4} \mathrm{~Pa}$, accelerating voltage $8 \mathrm{kV}$, and sample current of $20 \mu \mathrm{A}$. The precise $\mathrm{Ar}^{+}$ion etching was used also to fabricate angle laps and to bare individual nanolayers and their interfaces.

Phonon characteristic energies were measured by high resolution electron energy loss spectroscopy (HREELS) at primary energies $10.0,14.0$, and $30.0 \mathrm{eV}$ in the inelastic impact mode. This technique records the spectrum $I(\Delta E)$ of electrons scattered by the sample surface, as a function of energy losses. The parameters of spectra acquisition were selected in the best way to provide high energy resolution of the spectrometer with a full width at half maximum (FWHM) of about 8-20 meV.

Auger electron microscopy (AEM) and electron microscopy of characteristic losses were used in the imaging of elements and plasmons intensity distribution, through the angle lap cross-section of multilayer coatings in individual layers and on interfaces at primary electron energy $E_{0}=$ $1450 \mathrm{eV}$. Resolution of images was $256 \times 256$ pixels at $\times 10000$ magnification. Topographic contrast was suppressed by image acquisition software.

The features of the layers' crystalline structure and interface were investigated by the electron energy loss fine structure (EELFS) method [12] close to the line of back-scattered electrons $\left(E_{0}=1450 \mathrm{eV}\right)$. Recently this technique (in this reflection mode) has been used for precise investigations of the atomic structure in thin surface layers [12]. Similarly to extended X-Ray absorption fine structure (EXAFS), the EELFS signal contains information about the local order structure of the nearest atomic neighbors at the surface. The method of mathematical spectra processing adopted in the EXAFS spectroscopy can be used to analyze the fine structure of electron spectra and allows determining the lengths of the atomic bonds, with results in good agreement with the crystallographic data. EELFS analysis was carried out in the range of $E_{0}+250 \mathrm{eV}$ close to the line of primary backscattering electrons $\left(E_{0}\right)$. Electron spectra were recorded at speed $0.4 \mathrm{eV} / \mathrm{s}$ in $N(E)$ mode at a constant analyzer energy with signal accumulation of 10 scans. Spectrometer settings were chosen to ensure the best energy resolution with good signal/noise ratio. This method provides information about the interatomic distances on the surface with an accuracy of about $0.02 \mathrm{~nm}$ in the nearest coordination spheres with radii of up to $0.7 \mathrm{~nm}$.

\section{Results and Discussion}

3.1. Micro- and Nanostructure of Multilayer Coatings. The multilayer coating has a total thickness of about $1.06 \mu \mathrm{m}$ and homogeneous structure with a uniform distribution of individual layers (Figure 1(a)). Higher magnification image at $\times 500 \mathrm{k}$ (b) shows that the multilayer structure is well defined with low intermixing and sharp interfaces.

According to GIXRD data (Figure 2), TiAlN layers in monolayer and multilayer coatings studied in this paper consisted mainly of hexagonal (h-TiAlN) nanocrystalline grains. This is evidenced by the different reflections as in wurtzite AlN but shifted to higher scattering angles due to the hexagonal lattice increase due to Ti incorporation. A detailed study of the bonding structure of monolithic h-TiAlN films was reported previously [13]. In addition, Ag layers had a nanocrystalline face centered cubic (fcc) structure with grain sizes depending on the layer thickness.

Lattice parameters of TiAlN and multilayer TiAlN/Ag crystalline lattices in coatings determined by GIXRD are presented in Table 1. They were used for calculations of radial distribution functions (RDF) in order to compare an averaged structure from diffraction data with a local one from EELFS. 


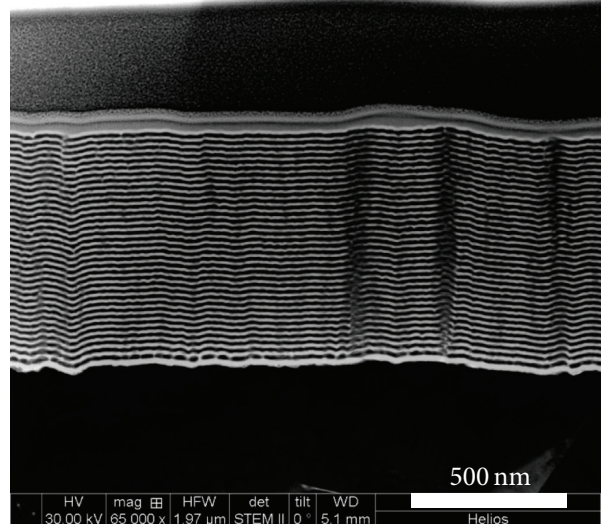

(a)

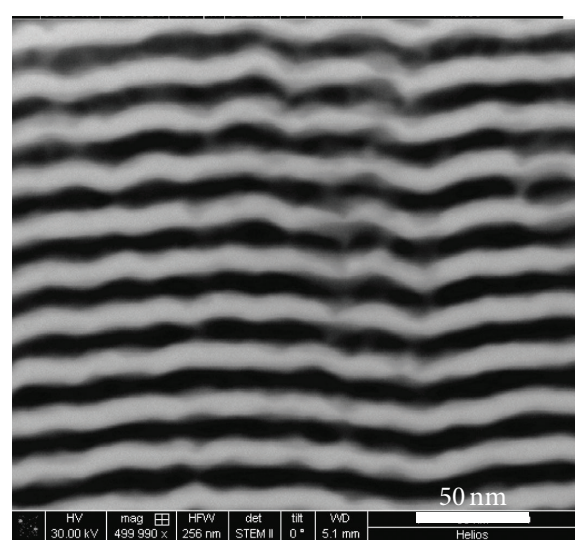

(b)

FIGURE 1: STEM bright field images of TiAlN/Ag multilayer coatings: (a) thickness of individual layers $15 \mathrm{~nm}, 35$ bilayers. Total coating thickness is $1065 \mathrm{~nm}$. (b) Detail of image with higher magnification.

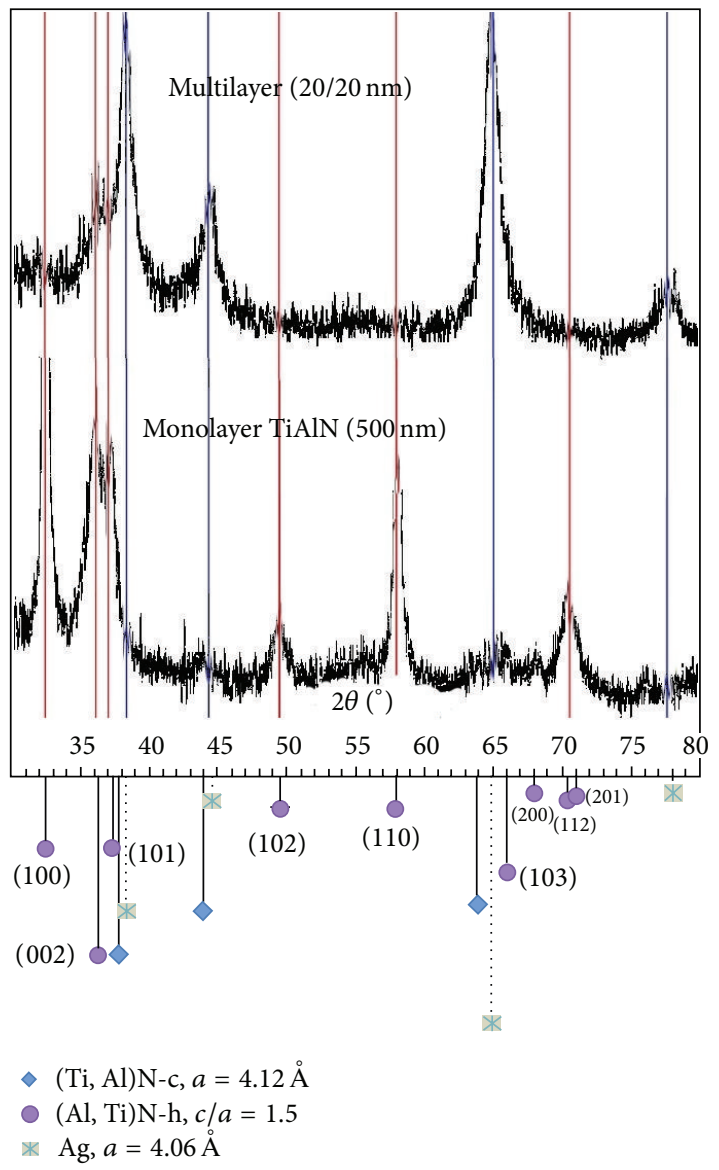

FIGURE 2: GIXRD analysis of TiAlN and TiAlN/Ag films grown by DC-MS

Figures 3 and 4 present Fourier transforms $[F(R)]$ of EELFS data for TiAlN and Ag layers in 20/20 nm nanocomposite and calculated RDFs based on GIXRD data. Peak positions in $F[R]$ correspond to coordination spheres radii in the crystalline lattice. The scheme of h-TiAlN unit cell
TABLE 1: Lattice parameters for h-TiAlN and Ag in the multilayer coating with $20 \mathrm{~nm} / 20 \mathrm{~nm}$ bilayers determined by GIXRD.

\begin{tabular}{lcccc}
\hline \multirow{2}{*}{ Layer composition } & Crystal lattice & \multicolumn{4}{c}{ Lattice parameters, $\AA$} \\
& $a$ & $b$ & $c$ \\
\hline TiAlN & $\begin{array}{c}\text { Hexagonal-wurtzite } \\
\text { type }\end{array}$ & 3.18 & 3.18 & 4.92 \\
$\mathrm{Ag}$ & FCC & 4.06 & 4.06 & 4.06 \\
\hline
\end{tabular}

based on EELFS data is shown in Figure 3(b). Our results on interatomic distances in the TiAlN nanolayer are in very good agreement with the ones measured by EXAFS for the nitride layer in TiAlN/Mo [14]. One can see (Figure 4) that $F(R)$ peaks are located at smaller distances compared to RDFs obtained from diffraction data.

The crystalline lattice of h-TiAlN layers in the nanolaminate coating with thin layers is strongly deformed compared to the thick monolayer coating. The lattice parameter " $a$ " is 0.86 times lower. Incorporation of $\mathrm{Al}$ in h-AlN deforms its lattice, lessening the parameter " $a$." Increase in the lattice parameter " $c$ " by incorporation of $\mathrm{Al}$ was also observed in [15]. Since we had the same composition of all nitride layers, this phenomenon is interpreted by features of nucleation and growth of nanolayers during their deposition.

Similar distortion of the crystalline lattice was also observed for Ag nanolayers. Main peaks on the $F(R)$ (Figure 4) are located at 1.45, 2.05, 2.70, and $3.95 \AA$. Interatomic distances of 1.45 and $2.05 \AA$ could be interpreted as $\mathrm{Ag}-\mathrm{N}$ bonds at the interfaces. Bulk Ag has fcc lattice with $a=4.085 \AA$ and radius of 1 st coordination sphere equal to $2.88 \AA$, so peaks at 2.70 and $3.95 \AA$ correspond to $\mathrm{Ag}-\mathrm{Ag}$ bonds in the fcc lattice with $a=3.95 \AA$. This means that the interatomic distance in the $\mathrm{Ag}$ nanolayer is 0.96 times lower than that in the bulk reference.

The best accommodation between growing nanolayers could be reached between Ag (111) and h-TiAlN (0001) planes where minimal interatomic distances $\mathrm{Ag}-\mathrm{Ag}(2.70 \AA)$ and $\mathrm{Ti}-\mathrm{Al}(2.73 \AA)$ misfit is approximately $1 \%$. During deposition 


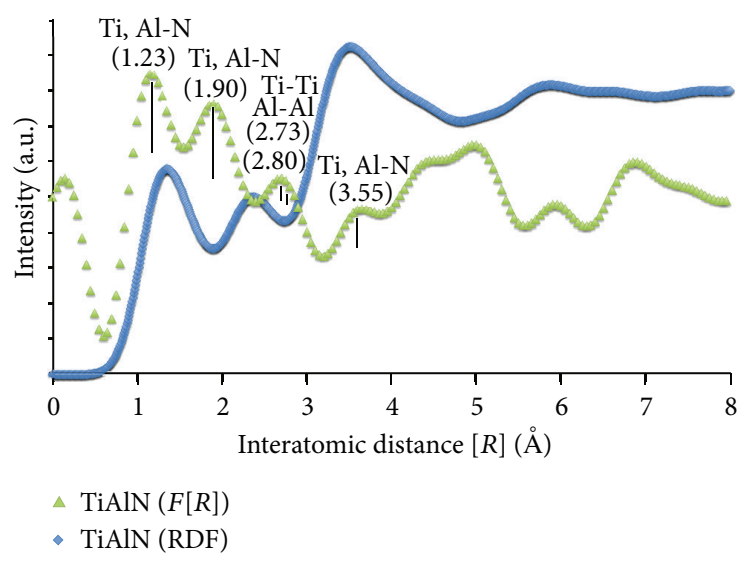

(a)

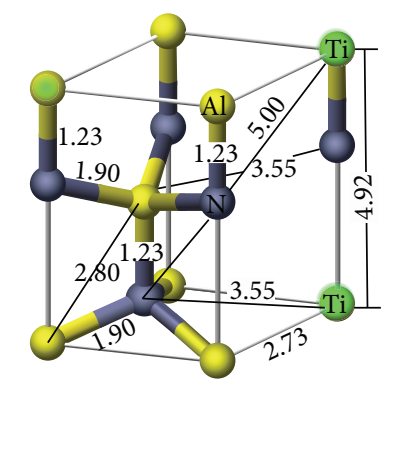

(b)

FIgURE 3: (a) Fourier transform $F[R]$ of EELFS from $20 \mathrm{~nm}$ TiAlN layer and calculated radial distribution function (RDF) from X-ray diffraction data; (b) scheme of nearest interatomic distances in h-TiAlN lattice.

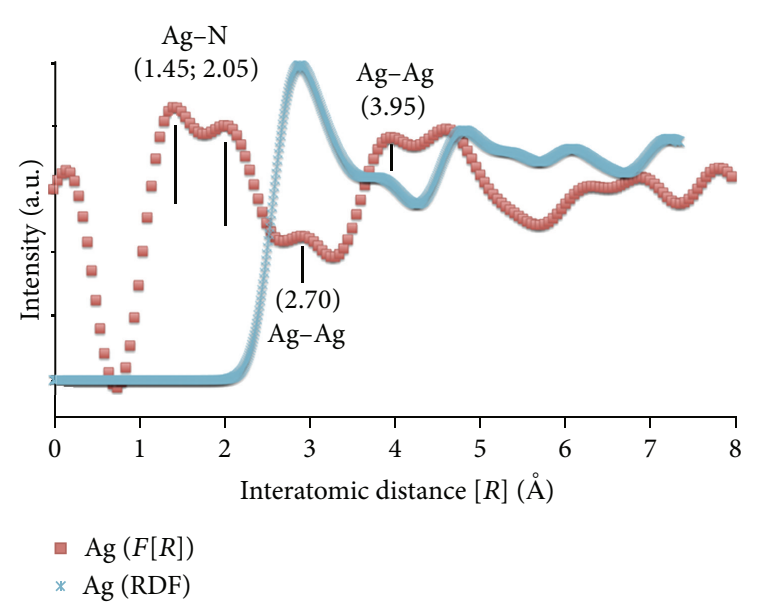

FIGURE 4: Fourier transform $F[R]$ of EELFS from $20 \mathrm{~nm}$ Ag layer and calculated RDF from $\mathrm{X}$-ray diffraction data.

of the coating, atoms of the growing film partly inherit the atomic arrangements of the underlayer. It is most likely that the metal-on-nitride and nitride-on-metal interfaces contain different amounts of semicoherent areas during deposition as the matrix phases are alternated.

\subsection{Transformations in Core Levels Electronic Structure Mea-} sured by X-Ray Photoelectron Spectroscopy (XPS). Figure 5 presents the series of XPS spectra from Ag, TiAlN/Ag interface, and (TiAl)N layers after $\mathrm{Ar}^{+}$ion depth profiling of multilayer nanolaminate coatings with 3 bilayers (3BL) and 35 bilayers (35BL). XPS was taken from central area of a plain etching crater formed by penning ion gun. Ion mixing and selective sputtering were excluded by the ion etching settings.

Binding energies of core level electrons are increased in the nanocrystalline state. Figure 6 demonstrates that $\mathrm{Ag} 3 \mathrm{~d}$ photoelectron lines are shifted to higher binding energies when the thickness of the Ag nanolayer decreases. These size dependent transformations of the nanomaterial electronic

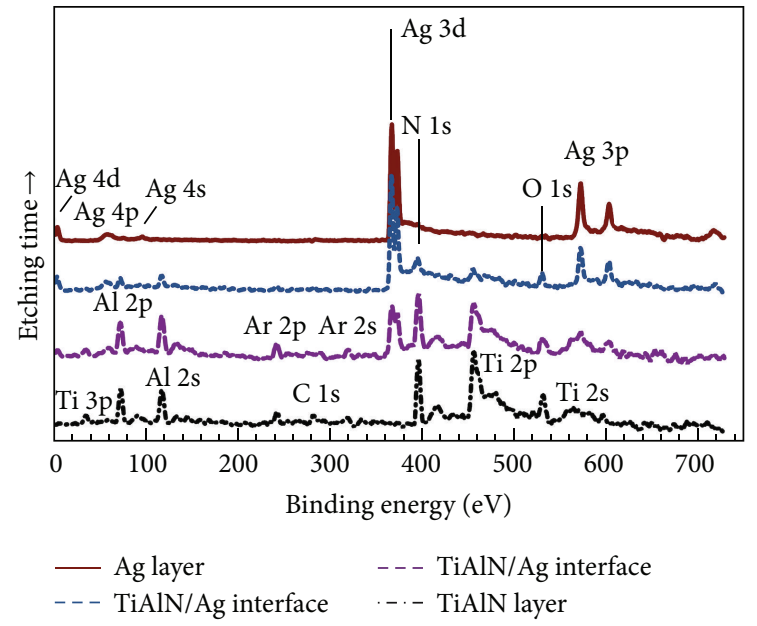

FIGURE 5: XPS spectra of (TiAl)N/Ag coating after $\mathrm{Ar}^{+}$ion etching: $\mathrm{Ag} 15 \mathrm{~nm}$ layer (top), interface with trace concentration of $\mathrm{Ag}$ on (TiAl) layer, and (TiAl)N layer (bottom).

structure are similar to that described in [16]. This size phenomenon is attenuated when depth profiling goes down to the Ag-TiAlN interface due to passivation of dangling bonds and partial coherence for the boundaries of Ag islands in individual layers.

The dependence of size shift on Ag layers thickness is plotted in Figure 7. The same phenomena were observed in many other cases of semiconductor nanocrystals with different shapes on the surface or embedded in dielectric matrixes [17-19]. The critical thickness of the Ag layer is near $D_{q}=$ $23.5 \mathrm{~nm}$ and, hence, physical properties of thinner nanocomposite layers are controlled mostly by quantum effects.

Transformations in Ag 5s and 4d electronic levels with a decrease of Ag nanolayer thickness are shown in Figure 8. The plots show that nanostructuring changes also the intensities and binding energies of Ag atoms outer shells. The Fermi level measured as a position of $\mathrm{Ag} 5$ s line moves by approximately 


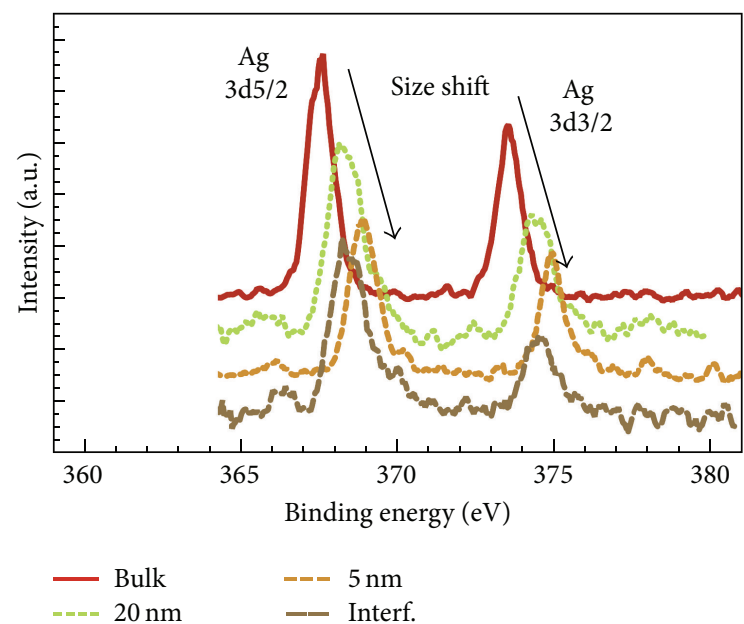

FIgURE 6: Ag 3d XPS spectra from nanocrystalline layers in (TiAl)N/Ag coatings with 3 (corresponds to bulk Ag), 6, and 35 bilayers.

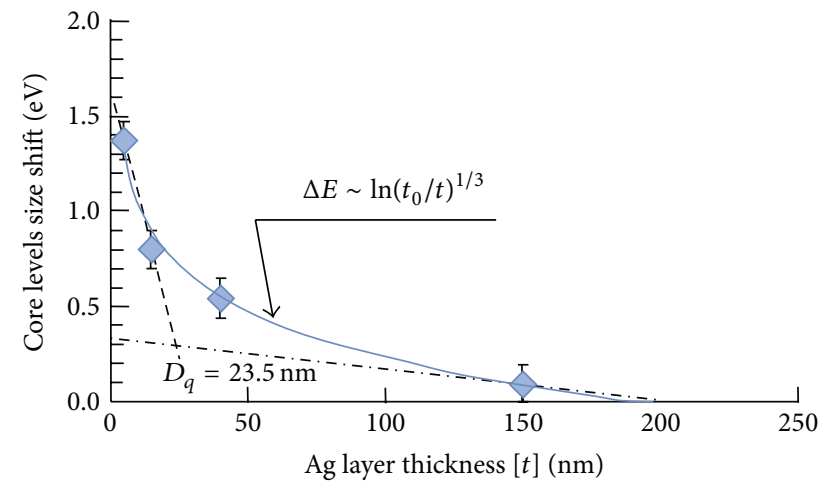

FIGURE 7: Size shift of XPS Ag 3d binding energy versus Ag layer thickness.

$1.8 \mathrm{eV}$ deeper into the 10 and $20 \mathrm{~nm}$ Ag films compared with the reference and the $150 \mathrm{~nm}$ layer. The intensity of this line grows inversely to the intensity of the $\mathrm{Ag} 4 \mathrm{~d}$ doublet, which goes down with the reduction of layer thickness. Also an increase in the spin-orbital splitting is observed. This redistribution of the outer level peak intensities is caused by the growth of electron concentration below the Fermi level. Correspondingly, metallic interatomic bonds in thin silver layers became more covalent. These results are compatible with data presented in [20] where transformations in $\mathrm{Ag} 5 \mathrm{~s}$ and $4 \mathrm{~d}$ levels were studied for very thin (2-3 monolayers) films. Applying the geometric boundaries on Ag layer results in the decreasing of probability of transition from valence band to continuum. This could be seen like shifting of Ag $5 \mathrm{~s}$ lines and increasing of their intensity or, in other words, as accumulation of electrons at the bottom of Fermi level without their ejection to conductive states. The splitting of $4 \mathrm{~d}$ electronic states was increased with layer thickening. These splitting changes are widely known for nanocrystals and the redistribution of intensities could be connected to a decrease in the proportion between volume/surface states.

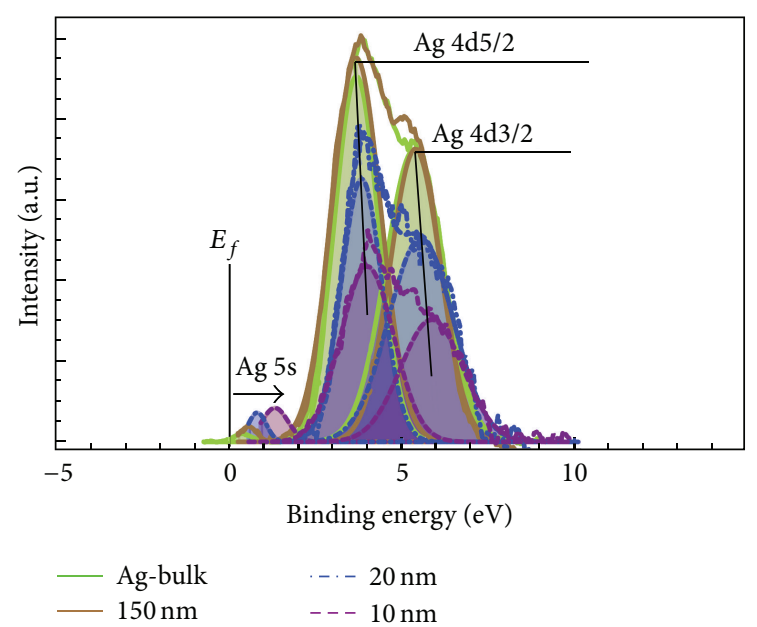

Figure 8: Ag 5s and 4d photoelectron lines for Ag standard and Ag layers in multilayer coatings.

3.3. Features of Plasmonic Structure Measured by EELS Scanning Microscopy. The sharp modifications of the electronic structure as the layer thickness is reduced have an impact on the plasmon oscillation distribution. We fulfilled detailed investigations of plasmon structure features in nanocomposites with $\mathrm{Ag}$ layers thickness lower than the determined critical size of $23.5 \mathrm{~nm}$, that is, in the TiAlN/Ag 20/20 $\mathrm{nm} \times$ 12 bilayers and $20 / 5 \mathrm{~nm} \times 33$ bilayers.

Figure 9 presents the backscattered electrons image (BEI) obtained from the TiAlN/Ag multilayer coating angle lap and Auger lines profiles along 1-1 direction using scanning Auger microscopy in characteristic Auger signals at energies of $351.0 \mathrm{eV}$ (Ag MNN) and $382.0 \mathrm{eV}$ (N KLL + Ti LMM). There we can see $4 \mathrm{TiAlN} / \mathrm{Ag}$ bilayers. High depth resolution has been achieved through the investigation of low angle (about $0.05^{\circ}$ ) cross-section of the nanocomposite.

Low-range EELS measurements were carried out with the primary energy $E_{0}=1450 \mathrm{eV}$. Energy losses spectra were acquired from sets of points on these angle laps surface at $\times 2000$ magnification. Each spectrum was normalized on the intensity of back scattered electron peak. Ten low-loss spectra were recorded from the central area of the layers and from the interfaces. Since the data acquisition took a considerable time $(30 \mathrm{~min})$, the electron beam current and energy were precisely stabilized. Charge neutralization by low energy electrons with $E_{0}=30.0 \mathrm{eV}$ was used. Figure 10 shows four normalized low-loss spectra with subtracted primary peak highlighting the influence of atomic composition (matrix effect) on the intensities of spectra fine structure. Peaks at $3.7 \mathrm{eV}$ correspond to surface plasmon excitation [21] and peaks at $7.7 \mathrm{eV}$ are associated with bulk plasmons. Good correlation between different surface and bulk plasmon features are observed on these spectra. The intensity of plasmon losses in the TiAlN dielectric layer was extremely low.

The surface and bulk plasmon loss energies from interfaces are shifted compared to their positions in the center of the Ag layer. Moreover, we can assume that the two different surface plasmons are excited from top and bottom sides of 


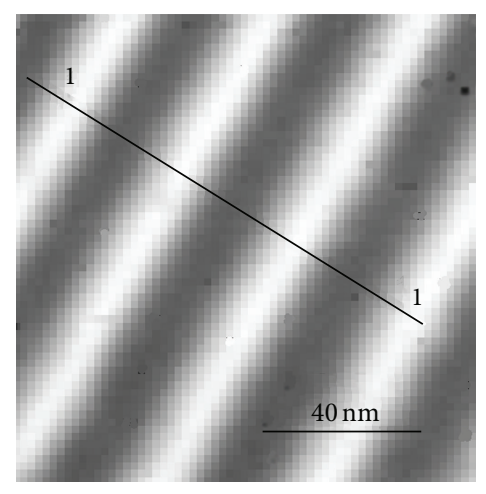

(a)

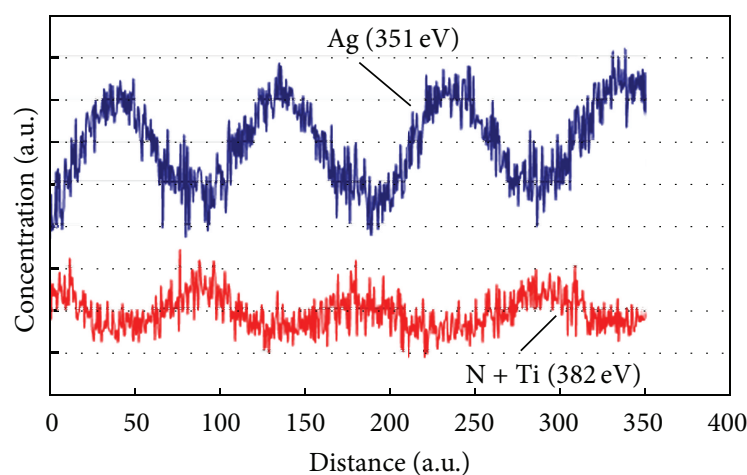

(b)

FIGURE 9: Structure of TiAlN/Ag 20/20 nm multilayer metamaterial: (a) back scattered electrons image (BEI) of angle lap at magnification $\times 2000$ (the scale relates to perpendicular cross-section presented in Figure 1); (b) chemical composition profile obtained by Auger electron spectroscopy along the 1-1 line in (a).

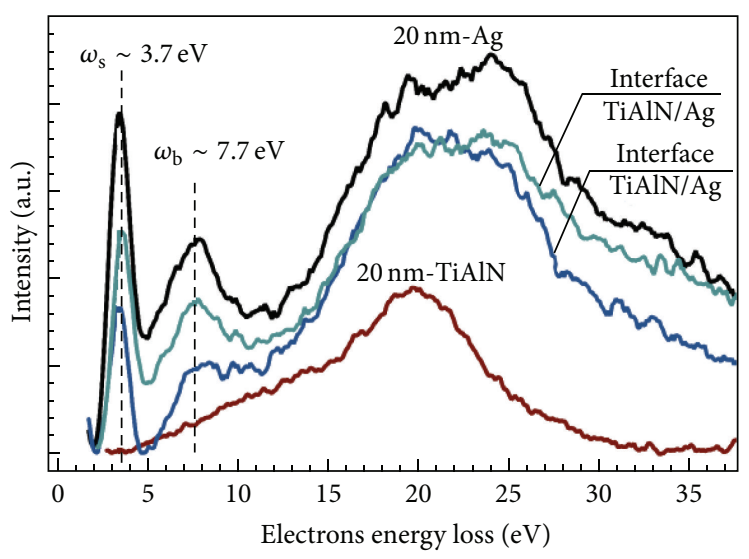

FIGURE 10: EELS from individual layers and interfaces of the multilayer TiAlN/Ag 20/20 nm coatings after normalizing of intensities and subtracting back scattered electron peak.

the Ag film. Precise determination of their energies has allowed us to study their distribution in the multilayer composite.

As one can see in Figures 11(b) and 11(c), surface plasmons with $\Delta E=4.0 \mathrm{eV}$ are excited in the relatively thin band comparing to $\mathrm{Ag}$ layer thickness along the top interfaces formed by nitride grown on the silver layer. Another surface plasmon with lower $\Delta E=2.0 \mathrm{eV}$ (Figure 11(c)) is localized at the bottom side of the silver nanolayer. These distinctions of electronic structure near the interfaces are controlled by variations in phase accommodation and perfection of lattice coherence as well as quantum effects. This results in the splitting of conducting electron states in thin Ag films. Bulk plasmons are excited at $\Delta E=7.7 \mathrm{eV}$ with maximal intensity in the central area of Ag layers.

Decreasing the metal layer thickness makes strong changes in the plasmon oscillations localized near the interface between Ag and TiAlN. Figure 12 was obtained while studying a nanocomposite with $5 \mathrm{~nm}$ Ag layers surrounded by $20 \mathrm{~nm}$ TiAlN. The BEI (Figure 12(a)) is obtained at primary energy $E_{0}=1417.6 \mathrm{eV}$. Image in Figure 12(b) shows the distribution of surface plasmon resonance occurring at energy loss of $3.0 \mathrm{eV}\left(E_{\mathrm{kin}}=1414.6 \mathrm{eV}\right)$ over the angle lap. This image differs significantly from the one presented in Figure 11(b) for the sample with $20 \mathrm{~nm}$ silver layers. Surface plasmons in the $5 \mathrm{~nm}$ Ag layer are distributed symmetrically near the layer boundaries. This may be explained since, as the thickness of the Ag interlayer is decreased from $20 \mathrm{~nm}$ to $5 \mathrm{~nm}$, the gradient of the magnetic field strength transverse component $H_{y}$ is changed through the film cross-section. That is, the antisymmetric $H_{y}$ field mode transforms to a symmetric $H_{y}$ field mode [11].

Figure 13 presents the fine comparison of surface plasmon $\left(\omega_{\text {sp }}\right)$ energies for $5 \mathrm{~nm}$ and $20 \mathrm{~nm} \mathrm{Ag}$ interlayers in TiAlN dielectric surrounding. One can see that the energy and intensity of surface plasmons are decreased with the thinning of silver layers. The relation of the surface to bulk plasmon intensities $I\left(\omega_{\mathrm{sp}}\right) / I\left(\omega_{\mathrm{b}}\right)$ goes down from 2.1 in the $20 \mathrm{~nm} \mathrm{Ag}$ layer to 1.7 in the $5 \mathrm{~nm}$ layer. 


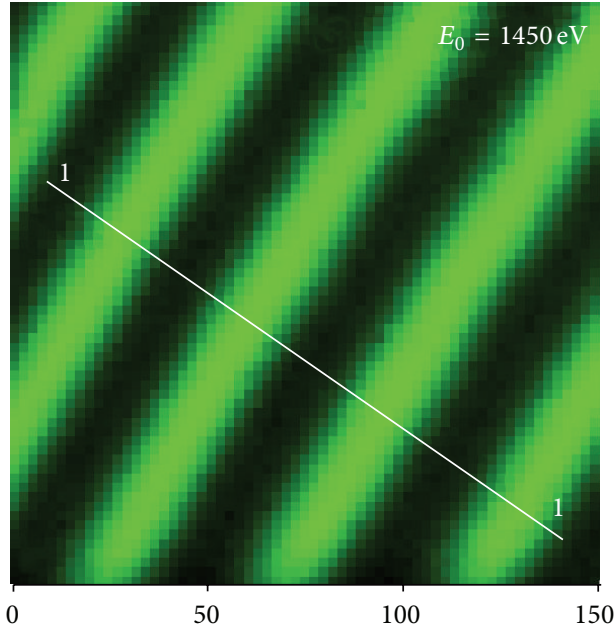

(a)

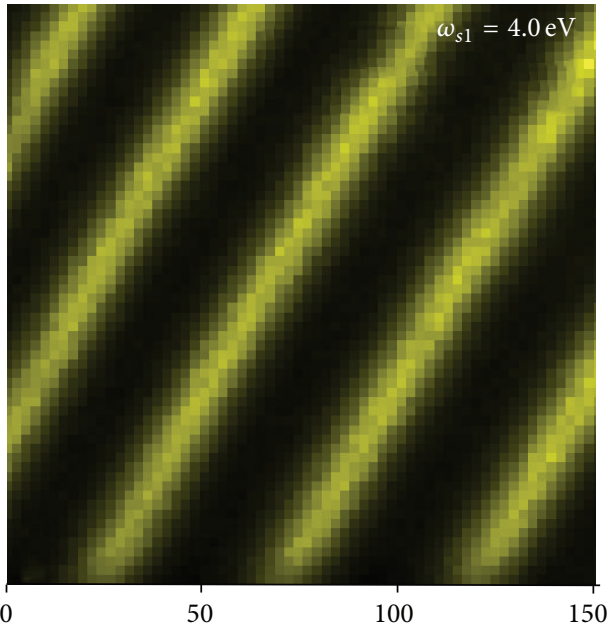

(b)

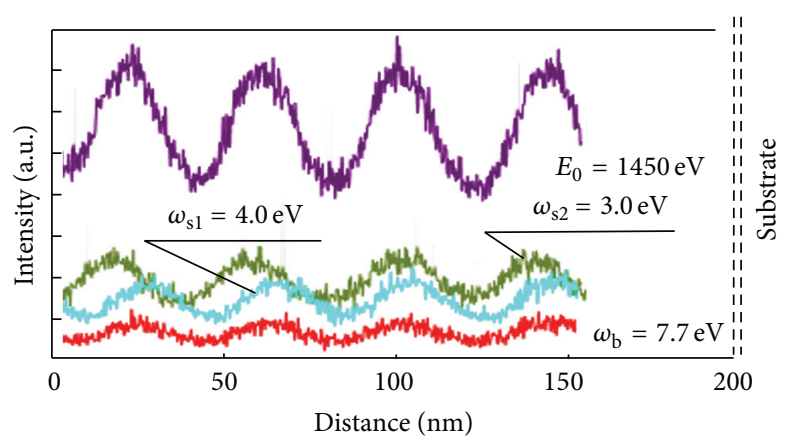

(c)

FIGURE 11: Structure of TiAlN/Ag 20/5 nm multilayer metamaterial: (a) BEI $\left(E_{0}=1450 \mathrm{eV}\right)$ of angle lap at magnification $\times 2000$; (b) plasmon distribution image at energy loss $4.0 \mathrm{eV}$; (c) profiles of back scattered electrons, surface, and bulk plasmon losses peak intensities along direction 1-1.

Nanostructuring of metal layers seems to cause the increase of surface and interface states' impact on metallic properties. When the metal layer is thinned below the plasmon wavelength, the plasmons are localized symmetrically along the metal-nitride interface. The observed alteration of plasmon distribution mechanism allows us to assume radical transformations of the nanocomposite wave properties in these boundary conditions. As quantum size phenomena stipulate electromagnetic properties of a medium below a critical size of $23.5 \mathrm{~nm}$, they also determine the conditions of resonance and propagation of plasmons in this artificial space.

These results allow us to suppose that transformations of plasmons phase space and interference in metamaterials could be considered in accordance with the variation of structural elements size.

\section{Conclusions}

The electronic structure of silver in the nanolaminate coating is starkly different from bulk reference material. Significant transformations are observed at a thickness of less than $23.5 \mathrm{~nm}$ due to quantum effects prevailing over those indicating "classic" growth of surface atomic influence.
Localization of electronic states in outer levels of $\mathrm{Ag}$ is growing alongside with the thinning of $\mathrm{Ag}$ layers in the TiAlN/Ag nanolaminate composite. This localization reduces the exchange of electrons between valence and conducting bands and consequently the concentration of charge carriers in the conducting band.

Significant splitting of the wave properties of plasmons is observed in the multilayer metal-dielectric material under investigation that is functioning as a plasmonic metamaterial with plasmon propagation dependent on the perfection of interfaces and layers constraint (or volume/surface ratio in thin individual layer).

\section{Conflict of Interests}

The authors declare that there is no conflict of interests regarding the publication of this paper.

\section{Acknowledgments}

This research was partially supported by RFBR Research Project no. 14-08-00472_a, RSF Research Project no. 14-1200170, and Russian Federation President Scholarship no. 


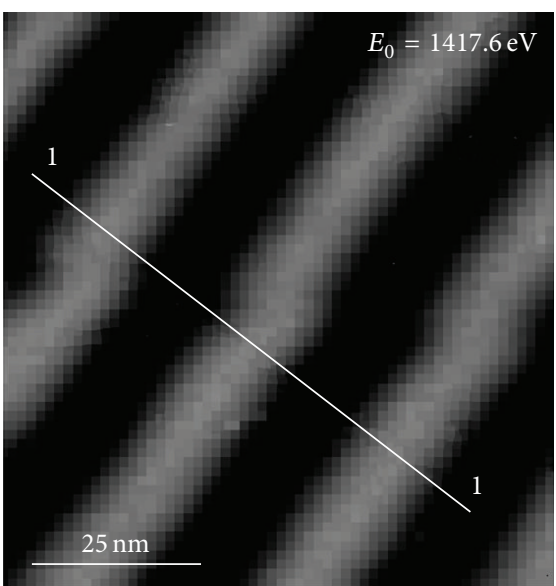

(a)

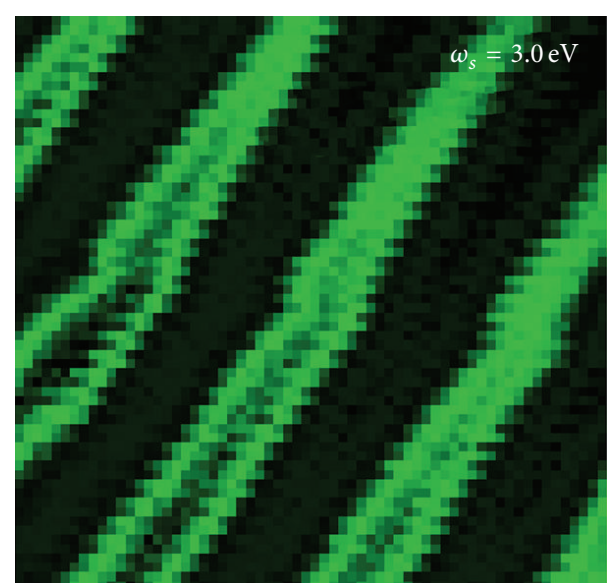

(b)

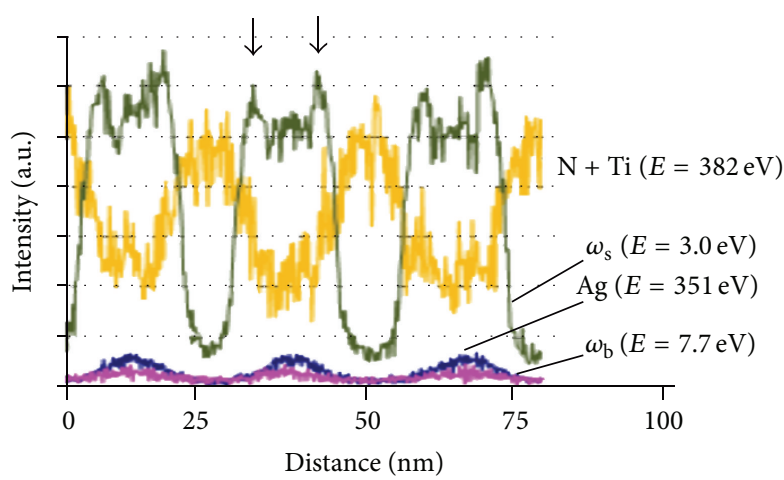

(c)

Figure 12: Structure of TiAlN/Ag $(20 / 5 \mathrm{~nm}):\left(\right.$ a) BEI $\left(E_{0}=1417.6 \mathrm{eV}\right)$ of angle lap at magnification $\times 2000$; (b) plasmon image at $\Delta E=3.0 \mathrm{eV}$; (c) profiles of signal intensities from $\mathrm{Ag}(351.0 \mathrm{eV})$ and $\mathrm{N}+\mathrm{Ti}(382.0 \mathrm{eV})$ Auger lines, surface $\left(\omega_{\mathrm{s}}\right)$, and bulk $\left(\omega_{\mathrm{b}}\right)$ plasmon losses along direction 1-1. Arrows are marking symmetric distribution of bulk plasmons intensities in the Ag layer.

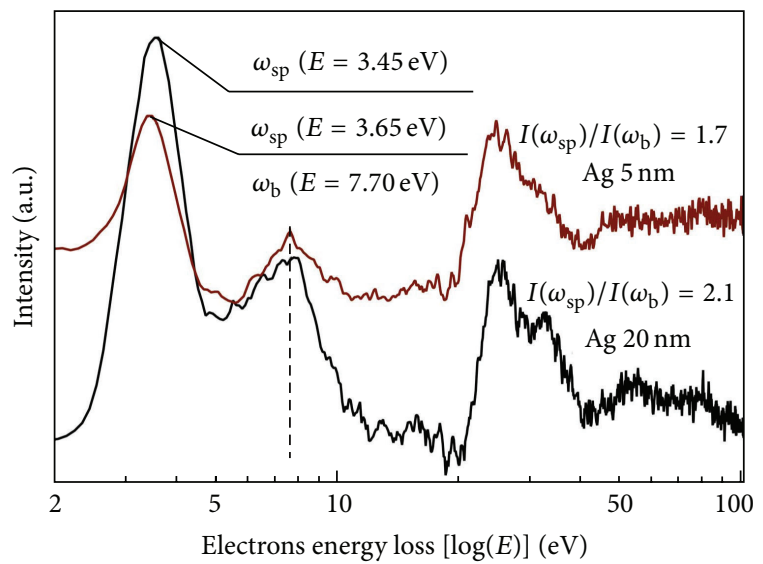

FIGURE 13: EELS of Ag interlayers in TiAlN/Ag multilayer coatings $\left(E_{0}=1450 \mathrm{eV}\right)$.

2040.2012.1. Additional support from Grant FIS2012-38866C05-05 (Ministerio de Economía y Competitividad, Spain) is also acknowledged. F. Soldera acknowledges the EFRE Funds of the European Commission for support of activities within the AME-Lab project.

\section{References}

[1] U. Guler, A. Boltasseva, and V. M. Shalaev, "Refractory plasmonics," Science, vol. 344, no. 6181, pp. 263-264, 2014.

[2] J. Anne Dionne, Flatland Photonics: Circumventing Diffraction with Planar Plasmonic Architectures [Thesis of Doctor of Philos$o p h y$ ], California Institute of Technology, Pasadena, Calif, USA, 2009.

[3] R. Marques, F. Martin, and M. Sorolla, Metamaterials with Negative Parameters: Theory, Design and Microwave Applications, Wiley Series in Microwave and Optical Engineering, WileyBlackwell, John Wiley \& Sons, 2008.

[4] A. Alù, A. Salandrino, and N. Engheta, "Negative effective permeability and left-handed materials at optical frequencies," Optics Express, vol. 14, no. 4, pp. 1557-1567, 2006.

[5] H. Shin and S. Fan, "All-angle negative refraction for surface plasmon waves using a metal-dielectric-metal structure," Physical Review Letters, vol. 96, Article ID 073907, 2006. 
[6] G. Shvets, "Photonic approach to making a material with a negative index of refraction," Physical Review B-Condensed Matter and Materials Physics, vol. 67, no. 3, Article ID 035109, 2003.

[7] M. I. Stockman, "Criterion for negative refraction with low optical losses from a fundamental principle of causality," Physical Review Letters, vol. 98, no. 17, Article ID 177404, 2007.

[8] A. Karalis, E. Lidorikis, M. Ibanescu, J. D. Joannopoulos, and M. Soljačić, "Surface-plasmon-assisted guiding of broadband slow and subwavelength light in air," Physical Review Letters, vol. 95, no. 6, Article ID 063901, 2005.

[9] X. Fan, G. P. Wang, J. C. W. Lee, and C. T. Chan, "All-angle broadband negative refraction of metal waveguide arrays in the visible range: theoretical analysis and numerical demonstration," Physical Review Letters, vol. 97, no. 7, Article ID 073901, 2006.

[10] A. Kovalev, D. Wainstein, and A. Rashkovskiy, "Investigation of anomalous physical properties of multilayer nanolaminate (TiAl)N/Cu coatings by electron spectroscopy techniques," Surface and Interface Analysis, vol. 42, no. 6-7, pp. 1361-1363, 2010.

[11] J. A. Dionne, E. Verhagen, A. Polman, and H. A. Atwater, "Are negative index materials achievable with surface plasmon waveguides? A case study of three plasmonic geometries," Optics Express, vol. 16, no. 23, pp. 19001-19017, 2008.

[12] D. L. Wainstein and A. I. Kovalev, "Fine determination of interatomic distances on surface using extended energy-loss fine structure (EELFS) data: peculiarities of the technique," Surface and Interface Analysis, vol. 34, no. 1, pp. 230-233, 2002.

[13] R. Gago, F. Soldera, R. Hübner et al., "X-ray absorption nearedge structure of hexagonal ternary phases in sputter-deposited TiAlN films," Journal of Alloys and Compounds, vol. 561, pp. 8794, 2013.

[14] C. J. Tavares, L. Rebouta, J. P. Rivière et al., "Atomic environment and interfacial structural order of TiAlN/Mo multilayers," Surface \& Coatings Technology, vol. 187, no. 2-3, pp. 393-398, 2004.

[15] A. Kimura, M. Kawate, H. Hasegawa, and T. Suzuki, "Anisotropic lattice expansion and shrinkage of hexagonal TiAlN and CrAlN films," Surface and Coatings Technology, vol. 169-170, pp. 367-370, 2003.

[16] B. Balamurugan and T. Maruyama, "Inhomogeneous effect of particle size on core-level and valence-band electrons: sizedependent electronic structure of $\mathrm{Cu}_{3} \mathrm{~N}$ nanoparticles," Applied Physics Letters, vol. 89, no. 3, Article ID 033112, 2006.

[17] A. I. Kovalev, D. L. Wainstein, A. Y. Rashkovskiy, A. Osherov, and Y. Golan, "Size shift of XPS lines observed from PbS nanocrystals," Surface and Interface Analysis, vol. 42, no. 6-7, pp. 850-854, 2010.

[18] A. I. Kovalev, D. L. Wainshtein, A. Y. Rashkovskiy, Y. Golan, A. Osherov, and N. Ashkenazy, "Studying of quantum-size effects origination in semiconducting lead sulfide nanocrystals," Protection of Metals and Physical Chemistry of Surfaces, vol. 46, no. 6, pp. 633-638, 2010.

[19] A. Kovalev, D. Wainstein, A. Rashkovskiy, A. Osherov, Y. Golan, and N. Ashkenasy, "Complex investigation of electronic structure transformations in Lead Sulphide nanoparticles using a set of electron spectroscopy techniques," Vacuum, vol. 86, no. 6, pp. 638-642, 2012.
[20] J. Feydt, A. Elbe, H. Engelhard, G. Meister, and A. Goldmann, "Photoemission studies of the W(110)/Ag interface," Surface Science, vol. 452, no. 1-3, pp. 33-43, 2000.

[21] I. Lopez-Salido, N. Bertram, D. C. Lim, G. Gantefцr, and Y. D. Kim, "Experimental studies on plasmon resonance of $\mathrm{Ag}$ nanoparticles on highly ordered pyrolytic graphite (HOPG)," Bulletin of the Korean Chemical Society, vol. 27, no. 4, pp. 556$562,2006$. 

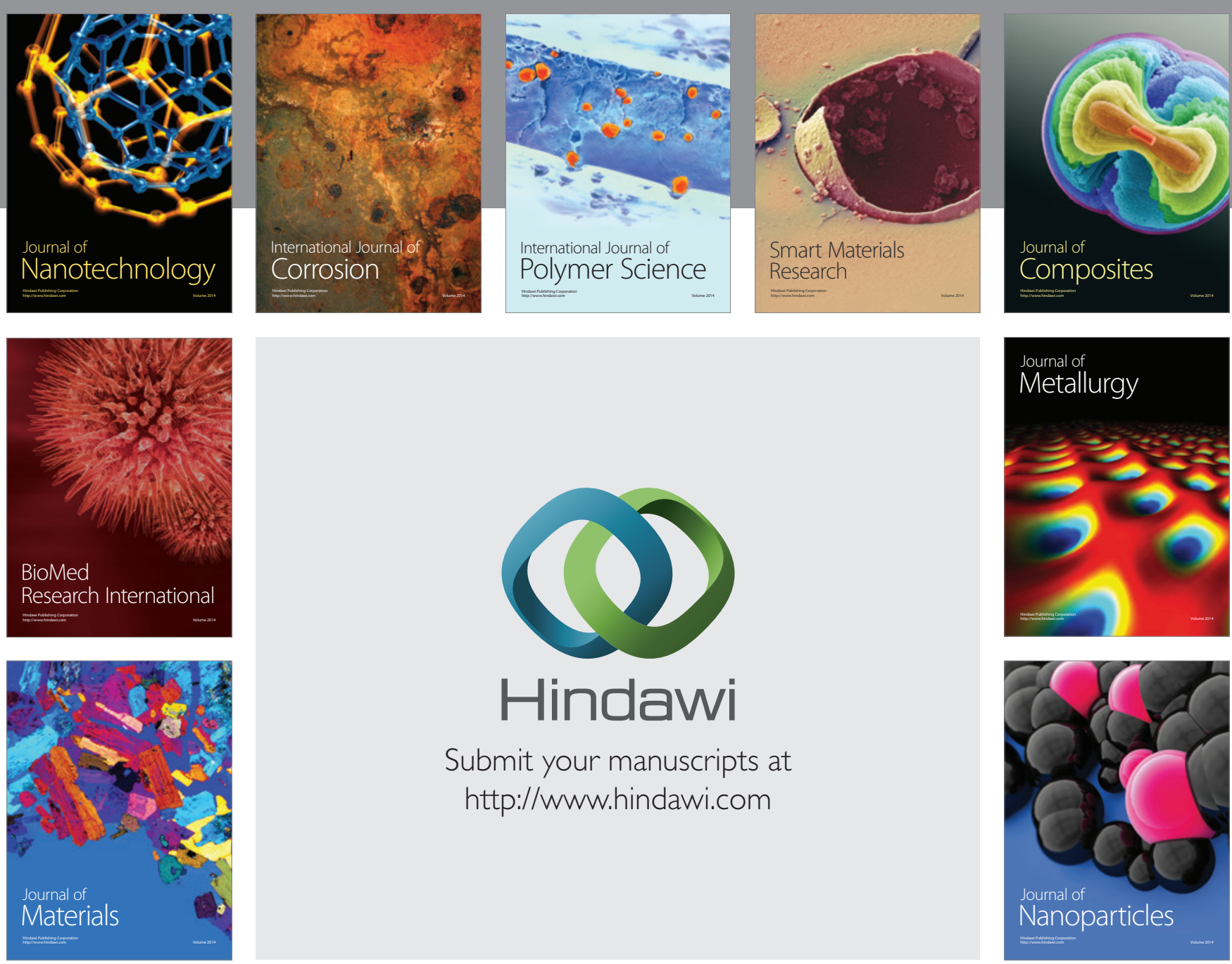

Submit your manuscripts at http://www.hindawi.com
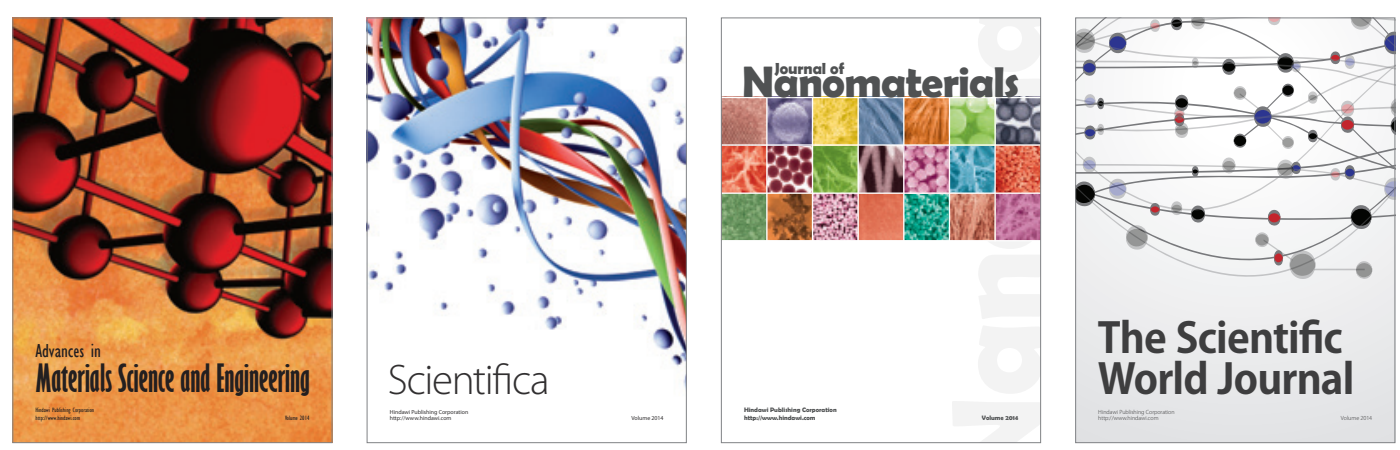

\section{The Scientific World Journal}
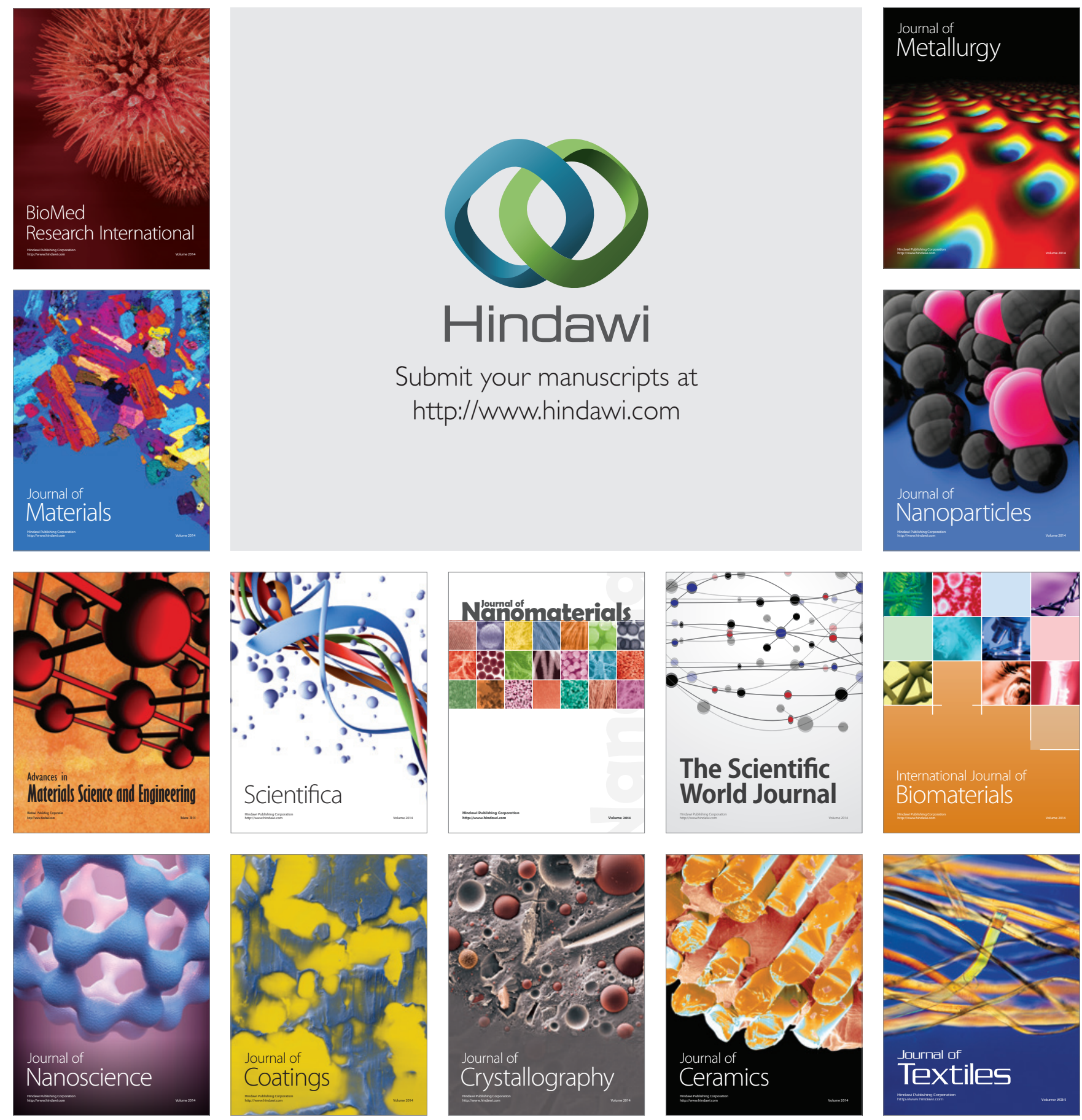